hep-ph/0110265, TIFR/TH/01-40

\title{
Quark number susceptibilities from lattice QCD*
}

\author{
Rajiv V. Gavai ${ }^{\dagger a}$ \\ a Department of Theoretical Physics, Tata Institute of Fundamental Research, \\ Homi Bhabha Road, Mumbai 400 005, India
}

Results from our recent investigations of quark number susceptibilities in both quenched and 2-flavour QCD are presented as a function of valence quark mass and temperature. A strong reduction $(\sim 40 \%)$ is seen in the strange quark susceptibility above $T_{c}$ in both cases. A comparison of our isospin susceptibility results with the corresponding weak coupling expansion reveals once again the non-perturbative nature of the plasma up to $3 T_{c}$. Evidence relating the susceptibility to another non-perturbative phenomena, pionic screening lengths, is presented.

\section{INTRODUCTION}

Investigations of quark number susceptibilities from first principles can have direct experimental consequences since quark flavours such as electric charge, strangeness or baryon number can provide diagnostic tools for the production of flavourless quark-gluon plasma in the central region of heavy ion collisions. As emphasized by B. Müller [3], and Koch 페 in this conference, fluctuations in conserved charges can act as probes of quark deconfinement since they are different in the hadronic and plasma phases in simple models. Indeed, excess strangeness production has been suggested as a signal of quarkgluon plasma almost two decades ago [5]. Lattice QCD can provide a very reliable and robust estimate for these quantities in both the phases since in thermal equilibrium they are related to corresponding susceptibilities by the fluctuation-dissipation theorem:

$$
\left\langle\delta Q^{2}\right\rangle \propto \frac{T}{V} \frac{\partial^{2} \log Z}{\partial \mu_{Q}^{2}}=\chi_{Q}\left(T, \mu_{Q}=0\right) .
$$

Here $\mu_{Q}$ is the chemical potential for a conserved charge $Q$, and $Z$ is the partition function of strongly interacting matter in volume $V$ at temperature $T$. Although Lattice QCD is unable to handle finite chemical potential satisfactorily at present, and cannot thus yield any reliable estimates of a number density, the susceptibility above, i.e., the first derivative of the number density at zero chemical potential, can be obtained reasonably well using the conventional simulation techniques.

Quark number susceptibilities also constitute an independent set of observables to probe whether quark-gluon plasma is weakly coupled in the temperature regime accessible to the current and future planned heavy ion experiments (say, $1 \leq T / T_{c} \leq 10$ ). A lot of the

\footnotetext{
${ }^{*}$ Based on work done [1,2] with Sourendu Gupta and Pushan Majumdar.
}

${ }^{\dagger}$ E-mail: gavai@tifr.res.in 
phenomenological analysis of the heavy ion collisions data is usually carried out assuming a weakly interacting plasma although many lattice QCD results suggest otherwise. It has been suggested [6] that resummations of the finite temperature perturbation theory may provide a bridge between phenomenology and the lattice QCD by explaining the lattice results starting from a few $T_{c}$. As we will see below, quark number susceptibilities can act as a cross-check of the various resummation schemes. Earlier work on susceptibilities [0] did not attempt to address this issue and were mostly restricted to temperatures very close to $T_{c}$. Furthermore, the quark mass was chosen there to vary with temperature linearly. We improve upon them by holding quark mass fixed in physical units $\left(m / T_{c}=\right.$ constant). We also cover a larger range of temperature up to $3 T_{c}$, and the accepted range of strange quark mass, in our simulations.

\section{FORMALISM}

After integrating the quarks out, the partition function $Z$ for QCD at finite temperature and density is given by

$$
Z=\int \mathcal{D} U \mathrm{e}^{-S_{g}} \operatorname{det} M\left(m_{u}, \mu_{u}\right) \operatorname{det} M\left(m_{d}, \mu_{d}\right) \operatorname{det} M\left(m_{s}, \mu_{s}\right) .
$$

Here $\left\{U_{\nu}(x)\right\}, \nu=0-3$, denote the gauge variables and $S_{g}$ is the gluon action, taken to be the standard Wilson action in our simulations. Since we employ staggered fermions, the Dirac matrices, $M$, are of dimensions $3 N_{s}^{3} N_{t}$, with $N_{s}\left(N_{t}\right)$ denoting the number of lattice sites in spatial(temporal) direction. $m_{f}$ and $\mu_{f}$ are quark mass and chemical potential (both in lattice units) for flavour $f$, denoting $u p(u)$, down $(\mathrm{d})$, and strange(s) above. The chemical potential needs to be introduced on lattice as a function $g(\mu)$ and $g(-\mu)$ multiplying the gauge variables in the positive and negative time directions respectively, such that [8] i) $g(\mu) \cdot g(-\mu)=1$ and ii) the correct continuum limit is ensured. While many such functions $g$ can be constructed, $\exp (\mu)$ being a popular choice, the results for susceptibilities at $\mu=0$ can easily be shown to be independent of the choice of $g$ even for finite lattice spacing $a$. From the $Z$ in eq. (2), the quark number densities and the corresponding susceptibilities are defined as

$$
n_{f} \equiv \frac{T}{V} \frac{\partial \ln Z}{\partial \mu_{f}} \quad \chi_{f f^{\prime}} \equiv \frac{\partial n_{f}}{\partial \mu_{f^{\prime}}}=\frac{T}{V}\left[\frac{1}{Z} \frac{\partial^{2} Z}{\partial \mu_{f} \partial \mu_{f^{\prime}}}-\frac{1}{Z} \frac{\partial Z}{\partial \mu_{f}} \frac{1}{Z} \frac{\partial Z}{\partial \mu_{f^{\prime}}}\right],
$$

To lighten the notation, we shall put only one subscript on the diagonal parts of $\chi$.

In order to obtain information for quark-gluon plasma in the central region, we evaluate the susceptibilities at the point $\mu_{f}=0$ for all $f$. In this case, each $n_{f}$ vanishes, a fact that we utilize as a check on our numerical evaluation. Moreover, the product of the single derivative terms in eq. (3) vanishes, since each is proportional to a number density. We set $m_{u}=m_{d}<m_{s}$. Noting that staggered quarks have four flavours by default, $N_{f}=4$, and defining $\mu_{3}=\mu_{u}-\mu_{d}$, one finds from eq. (3) that the isotriplet and strangeness susceptibilities are given by

$$
\chi_{3}=\frac{T}{2 V} \mathcal{O}_{1}\left(m_{u}\right), \quad \chi_{s}=\frac{T}{4 V}\left[\mathcal{O}_{1}\left(m_{s}\right)+\frac{1}{4} \mathcal{O}_{2}\left(m_{s}\right)\right]
$$

where $\mathcal{O}_{1}=\left\langle\operatorname{Tr}\left(M^{\prime \prime} M^{-1}-M^{\prime} M^{-1} M^{\prime} M^{-1}\right)\right\rangle, \mathcal{O}_{2}=\left\langle\left(\operatorname{Tr} M^{\prime} M^{-1}\right)^{2}\right\rangle, M^{\prime}=\partial M / \partial \mu$ and $M^{\prime \prime}=\partial^{2} M / \partial \mu^{2}$. The angular brackets denote averaging with respect to the $Z$ in eq. (2). 
One can similarly define baryon number and charge susceptibilities. We refer the reader for more details on them to Ref. [2].

In the discussion above, quark mass appears as an argument of $\mathcal{O}$ and implicitly in the Boltzmann factor of $Z$. Let us denote it by $m_{v a l}$ and $m_{\text {sea }}$ respectively. While the two should ideally be equal, we evaluated the expressions above in steps of improving approximations (and increasing computer costs) by first setting $m_{\text {sea }}=\infty$ for all flavours (quenched approximation [1]) and then simulating two light dynamical flavours, by setting $m_{\text {sea }} / T_{c}=0.1$ (2-flavour QCD [2]). In each case we varied $m_{\text {val }}$ over a wide range to cover both light $\mathrm{u}, \mathrm{d}$ quarks as well as the heavier strange quark. Details of our simulations as well as the technical information on how the thermal expectation values of $\mathcal{O}$ were evaluated are in Refs. [1, 2].

\section{RESULTS}

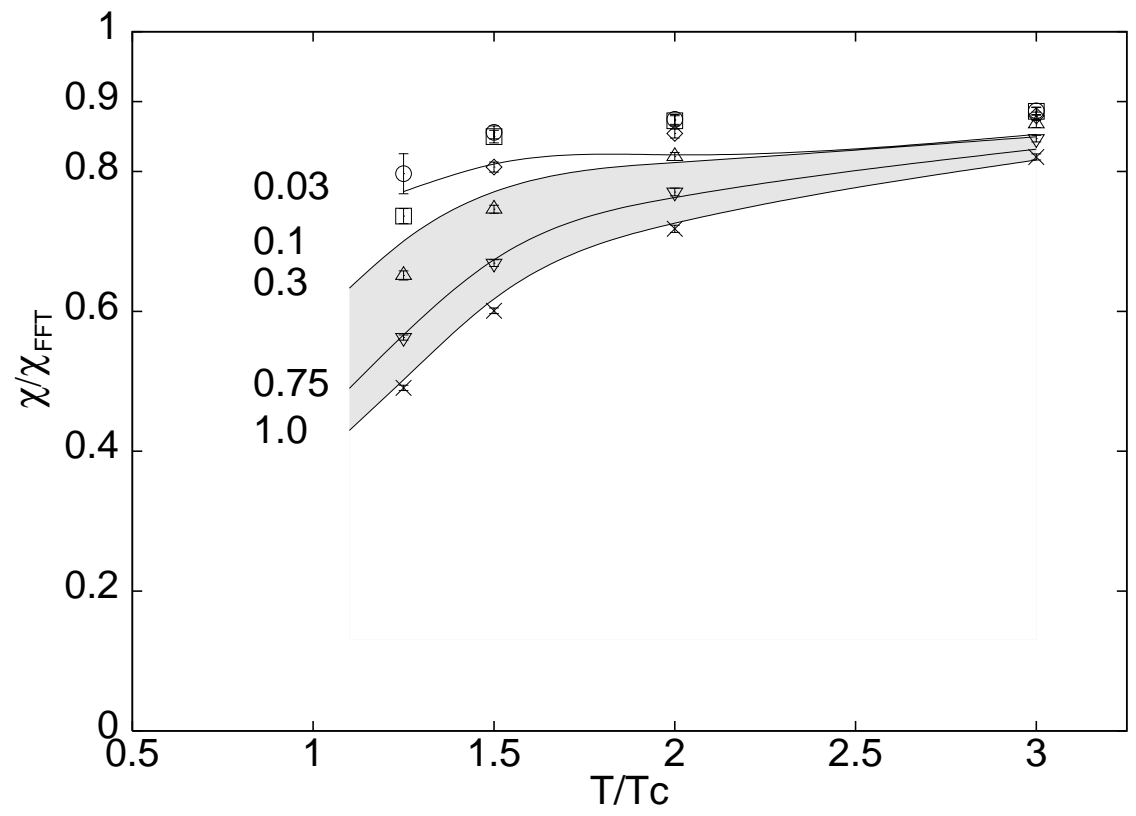

Figure 1. $\chi / \chi_{F F T}$ as a function of temperature for various valence quark masses.

Based on our tests [1] of volume dependence, made by varying $N_{s}$ from 8 to 16 , we chose $N_{s}=12$. Choosing $N_{t}=4$, and making simulations at the known $\beta_{c}\left(N_{t}^{\prime}\right)$ for $N_{t}^{\prime}=$ $6,8,12$, where $\beta_{c}$ is the gauge coupling at which chiral (deconfinement) transition/crossover takes place for lattices with temporal extent $N_{t}^{\prime}$, we obtained results at $T / T_{c}=1.5$, 2 and 3 in each case. The sea quark mass in our dynamical simulations corresponds to 14-17 MeV from the existing estimates [9] of $T_{c}$ for 2-flavour QCD. Fig. 11 shows our results, normalized to the free field values on the same size lattice, $\chi_{F F T}$, in the quenched approximation (interpolated continuous lines) and for 2 light flavours (data points) for a range of $m_{v a l} / T_{c}$ indicated on the left. Due to our choice of normalization, and the fact [2] that the contribution of $\mathcal{O}_{2}$ to $\chi_{s}$ turns out to be negligibly small for $T>T_{c}$, Fig. 11 
shows both $\chi_{3}$ and $\chi_{s}$.

Although $T_{c}$ differs in the quenched and 2-flavour QCD by a factor of 1.6-1.7, the respective susceptibilities in Fig. 目change by at most 5-10\% for each $m_{\text {val }}$. This suggests that making further the strange quark dynamical may not change the results significantly. Using a wide range for strange quark mass of 75 to $170 \mathrm{MeV}$, the strangeness susceptibility can be read off from the shaded region. It shows a strong temperature dependence and is smaller by about $40 \%$ compared to its ideal gas value near $T_{c}$. This has implications for phenomenology of particle abundances. Finally, the ratio $\chi / \chi_{F F T}$ is seen to be $0.88(0.85)$ for 2-flavour (quenched) QCD at the smallest $m_{\text {val }}$ and highest temperature we studied, with a mild temperature variation in its vicinity.

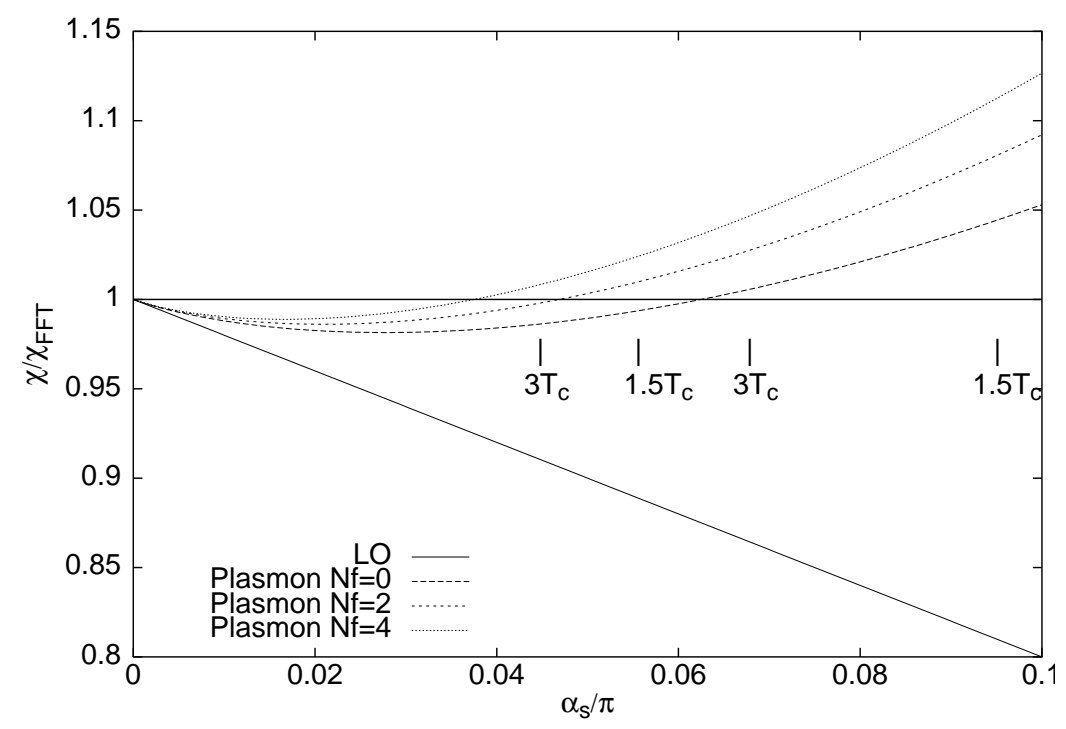

Figure 2. $\chi / \chi_{F F T}$ as a function of $\alpha_{s} / \pi$ for $N_{f}$ dynamical massless quarks.

Weak coupling expansion [10] yields $\chi / \chi_{F F T}=1-2 \frac{\alpha_{s}}{\pi}\left[1-4 \sqrt{\frac{\alpha_{s}}{\pi}\left(1+\frac{N_{f}}{6}\right)}\right]$. Fig. [2 shows the predictions for various $N_{f}$ along with the leading order $N_{f}$-independent prediction. Using a scale $2 \pi T$ for the running coupling and $T_{c} / \Lambda_{\overline{M S}}=0.49(1.15)$ for the $N_{f}=2(0)$ theory [9], the values $T / T_{c}=1.5$ and 3 are marked on the figure as the second (first) set. As one can read off from the figure, in both cases the ratio decreases with temperature in the range up to $3 T_{c}$ whereas our results in Fig. 1 display an increase. Furthermore, the perturbative results lie significantly above in each case, being in the range 1.027-1.08 for 2-flavour QCD and 0.986-0.994 for quenched QCD. This calls for clever resummations of perturbation theory or non-perturbative physics.

One known indicator of non-perturbative physics in the plasma phase is the screening length in the channel with quantum numbers of pion. While it exhibits chiral symmetry restoration above $T_{c}$ by being degenerate with the corresponding scalar screening length, its value is much smaller than the free field value unlike that for other screening lengths. Fig. 3 shows $\chi_{3}$ and $\chi_{\pi}$ (defined as a sum of the pion correlator over the entire lattice) as a function of $M_{\pi} / T$. It suggests the non-perturbative physics in the two cases to be closely related, if not identical. 


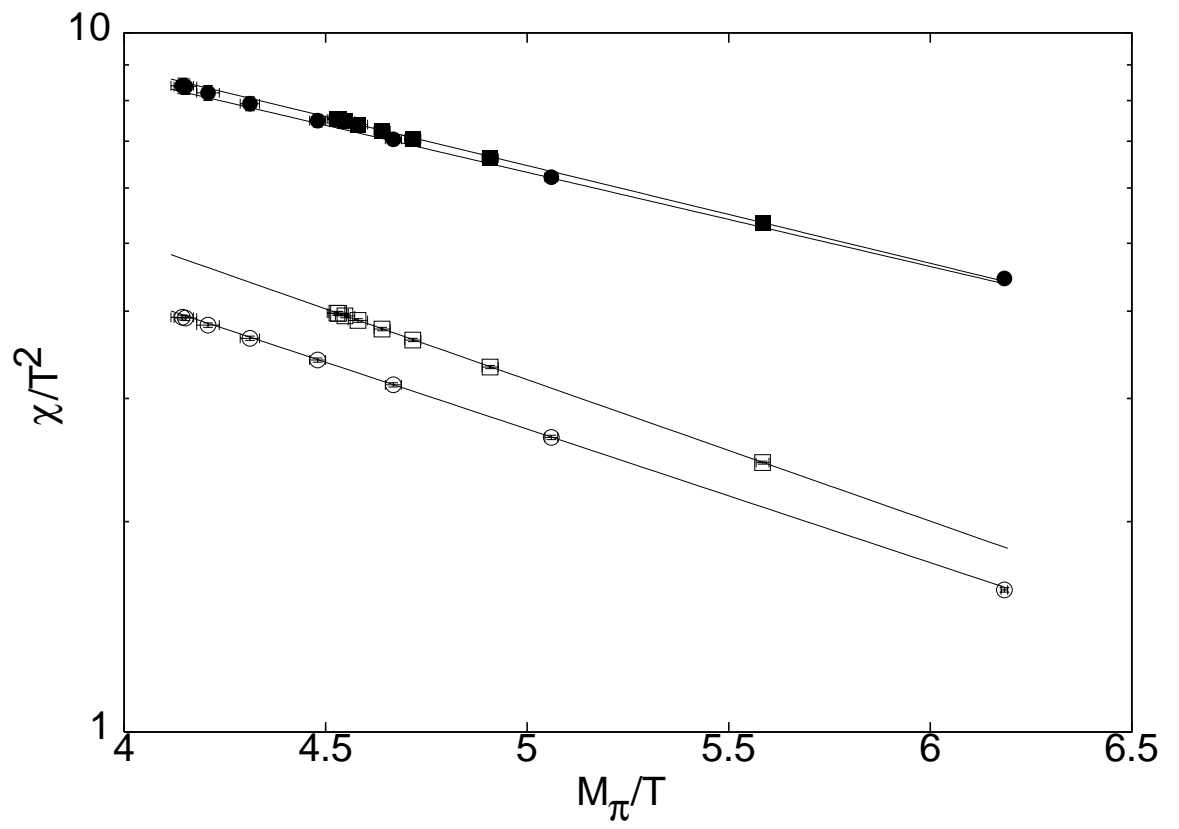

Figure 3. $4 \chi_{3} / T^{2}$ (open symbols) and $\chi_{\pi} / 10 T^{2}$ (filled symbols) as a function of $M_{\pi} / T$ at $2 T_{c}$ (circles) and $3 T_{c}$ (boxes).

\section{ACKNOWLEDGMENTS}

It is a pleasure to thank my collaborators Sourendu Gupta and Pushan Majumdar. I am grateful to the Alexander von Humboldt Foundation for its generous financial support which made my participation possible. It is a delight to acknowledge the warm hospitality of the Physics Department of the University of Bielefeld, especially that from Profs. Frithjof Karsch and Helmut Satz.

\section{REFERENCES}

1. R. V. Gavai and S. Gupta, Phys. Rev. D 64 (2001) 074506.

2. Rajiv V. Gavai, Sourendu Gupta and Pushan Majumdar, hep-lat/0110032.

3. B. Müller, in these proceedings; M. Asakawa, U. W. Heinz, and B. Müller, Phys. Rev. Lett. 85 (2000) 2072.

4. V. Koch, in these proceedings; S. Jeon and V. Koch, Phys. Rev. Lett. 85 (2000) 2076.

5. J. Rafelski and B. Müller, Phys. Rev. Lett. 46 (1982) 1066; erratum-ibid 56 (1986) 2334.

6. J. P. Blaizot et al., Phys. Rev. D 63 (2001) 065003; J. O. Andersen et al., Phys. Rev. D 63 (2001) 105008; K. Kajantie et al., Phys. Rev. Lett. 86 (2001) 10.

7. S. Gottlieb et al., Phys. Rev. Lett. 59 (1987) 1513; R. V. Gavai et al., Phys. Rev. D 40 (1989) 2743; S. Gottlieb et al., Phys. Rev. D 55 (1997) 6852.

8. R. V. Gavai, Phys. Rev. D 32 (1985) 519.

9. A. Ali Khan et al., Phys. Rev. D 63 (2001) 034502; F. Karsch et al., Nucl. Phys. B 605 (2001) 579; S. Gupta, Phys. Rev. D 64 (2001) 034507.

10. J. I. Kapusta, "Finite-temperature Field Theory", 1989, Cambridge University Press, Cambridge, UK, pp 132-133. 